\title{
Correction to: Sirt7-p21 Signaling Pathway Mediates Glucocorticoid-Induced Inhibition of Mouse Neural Stem Cell Proliferation
}

Mohammed A. H. Alnoud ${ }^{1}$ - Wen Chen ${ }^{1}$. Nana Liu ${ }^{1}$ - Wei Zhu ${ }^{1}$. Jing Qiao ${ }^{1}$ - Shujuan Chang ${ }^{1}$. Yukang Wu ${ }^{1}$. Shanshan Wang ${ }^{1} \cdot$ Yiwei Yang $^{1} \cdot$ Qiaoyi Sun ${ }^{1}$. Jiuhong Kang ${ }^{1}$

Published online: 20 April 2021

(c) Springer Science+Business Media, LLC, part of Springer Nature 2021

\section{Correction to: Neurotoxicity Research \\ https://link.springer.com/article/10.1007/ \\ s12640-020-00294-x}

The published Supplementary Fig. 1 was incorrect. Please see correct version below.

The original article can be found online at https://doi.org/10.1007/ s12640-020-00294-x.

Jiuhong Kang

jhkang@tongji.edu.cn

1 Clinical and Translational Research Center of Shanghai First

Maternity and Infant Hospital, Shanghai Key Laboratory

of Signaling and Disease Research, Collaborative Innovation

Center for Brain Science, School of Life Sciences

and Technology, Tongji University, 1239 Siping Road,

Shanghai 200092, China 

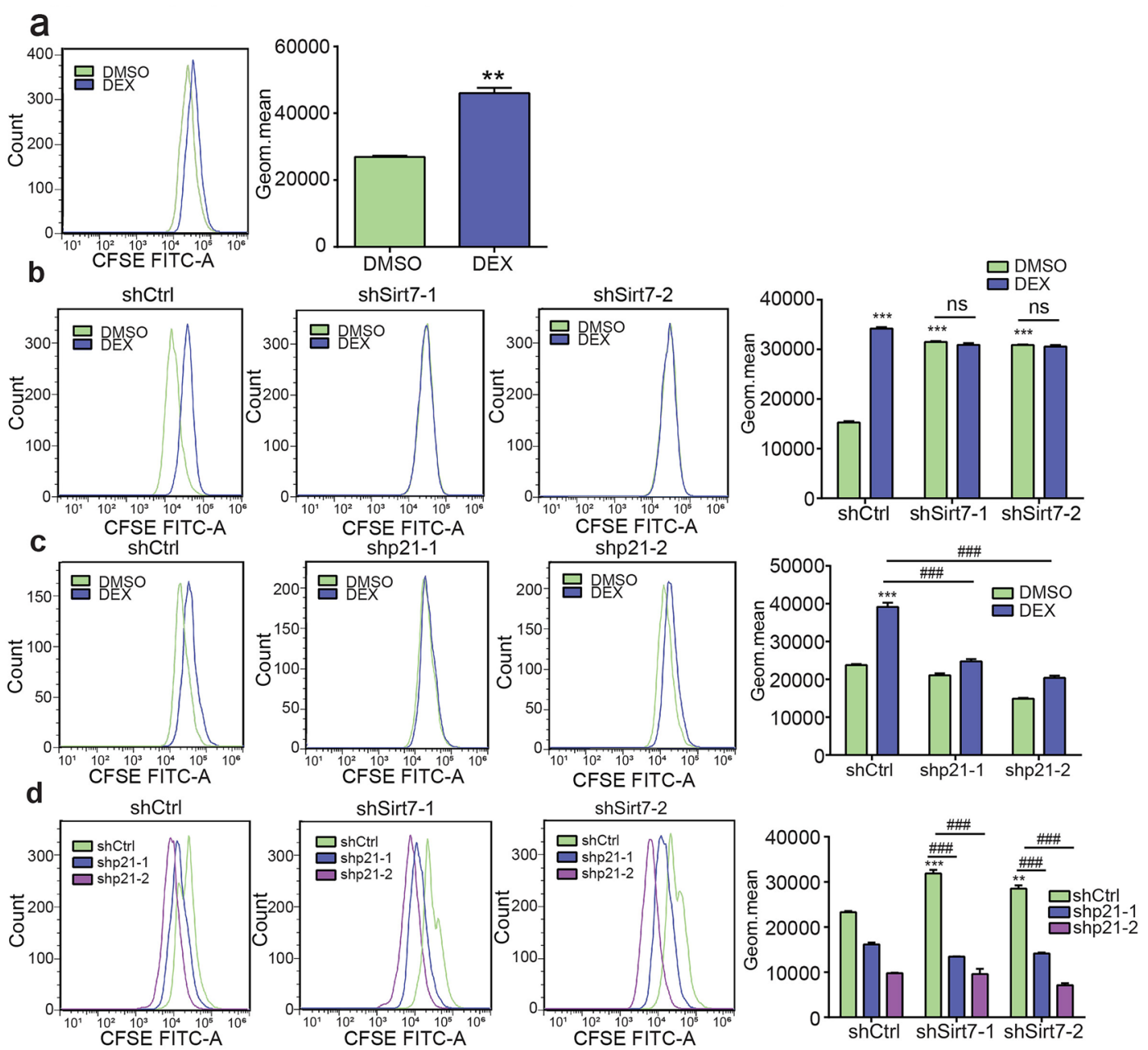

e 吕 HA-luc

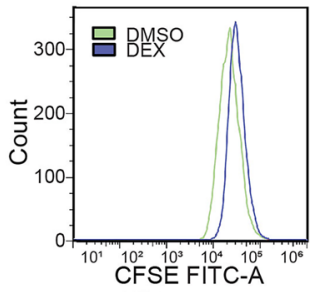

吕 HA-luc
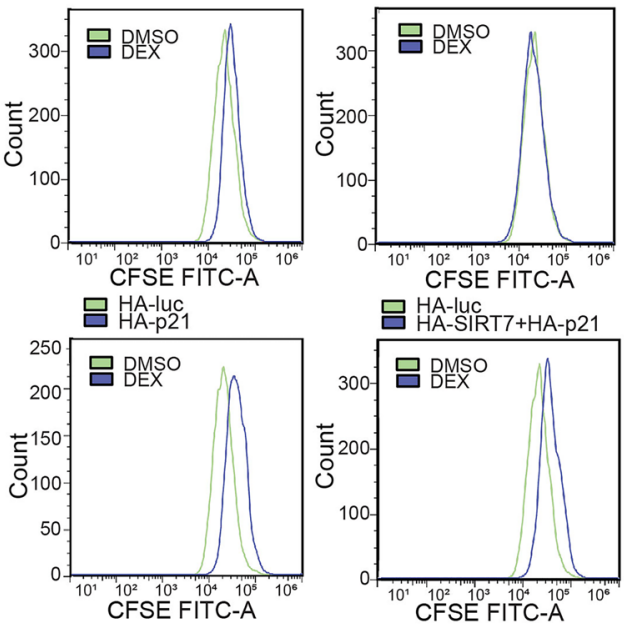

员 HA-luc
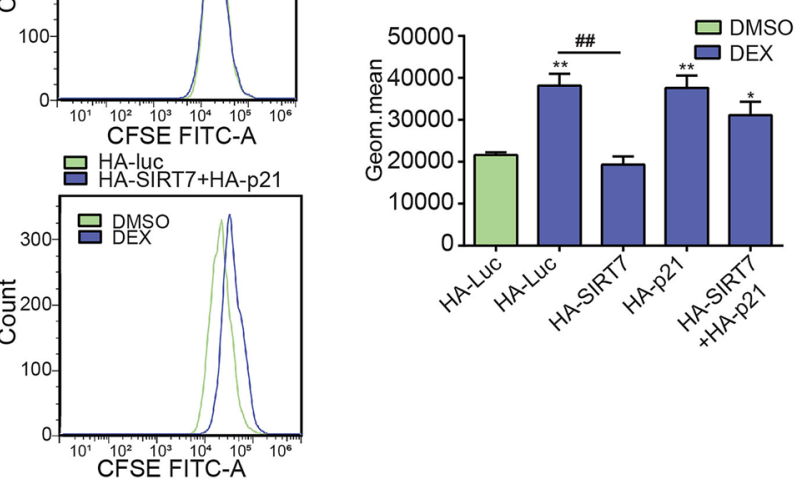

Supplementary Fig. 1 Test of NSC proliferation by CFSE based assay

Publisher's Note Springer Nature remains neutral with regard to jurisdictional claims in published maps and institutional affiliations. 